\title{
Using Mixed Methods to Adapt and Evaluate the Implementation of a Multicomponent Tobacco-Free Workplace Program within Behavioral Health Centers
}

Isabel Martinez Leal ( $\boldsymbol{\nabla}$ imarti31@central.uh.edu )

University of Houston https://orcid.org/0000-0002-0073-339X

\section{Tzu-An Chen}

University of Houston

Virmarie Correa-Fernández

University of Houston

Kathy Le

University of Houston

Daniel P. O'Connor

University of Houston

\section{Bryce Kyburz}

Integral Care

William T. Wilson

Integral Care

Teresa Williams

Integral Care

Lorraine R. Reitzel

University of Houston

\section{Research}

Keywords: tobacco control, behavioral health, mixed methods, users and researchers collaboration

Posted Date: March 23rd, 2020

DOI: https://doi.org/10.21203/rs.3.rs-18558/v1

License: (c) (i) This work is licensed under a Creative Commons Attribution 4.0 International License. Read Full License 


\section{Abstract}

Background: Behavioral health treatment centers (BHTCs) rarely implement existing evidence-based practices for treating tobacco dependence, despite high rates of tobacco use among their clients. Taking Texas Tobacco Free (TTTF) has successfully targeted this disparity by delivering an evidence-based, multilevel, tobacco-free workplace program providing policy implementation and enforcement, education, provider training in tobacco screenings and treatments, and nicotine replacement therapies (NRT) to BHTCs across Texas. We describe a mixed methods design used to conduct a formative evaluation process to adapt implementation strategies to local contexts, evaluate program outcomes and characterize processes influencing program implementation in two BHTCs serving 17 clinics.

Methods: Varied data collection included pre and post-implementation leader, provider, and staff surveys; and pre, mid, and post-implementation provider, staff and consumer focus groups. During implementation, data were collected via various logs (tobacco screenings, NRT delivery) to monitor program content delivery. The RE-AIM (Reach, Effectiveness, Adoption, Implementation, Maintenance) framework guided translation of behavioral interventions into sustainable practice.

Results: While program implementation varied between each BHTC, all clinics adopted a $100 \%$ tobacco-free workplace policy, integrated tobacco screenings into routine practice, delivered evidence-based interventions, dispensed NRT to consumers and staff, and increased provider knowledge on how to address tobacco dependence. Pre, mid, and post-implementation qualitative findings served to: 1) develop program strategies and materials adapted to local contexts and populations and address barriers; 2) adjust delivery systems of key components to enhance implementation; 3 ) understand reasons for success or failure to implement specific practices; and 4) reveal program integration into clinic culture, enhancing sustainability.

Conclusions: Implementation of TTTF at both BHTCs increased organizational capacity in the provision of evidence-based practices to treat tobacco dependence through successfully meeting most of our RE-AIM targets. Mixing methods involved program adopters and recipients as collaborators directly shaping core interventions to their individual context and needs, thus increasing program fit, ownership, adoption and sustainability; closing the gap between research and practice. These findings contribute to the development of flexible strategies and interventions capable of addressing variable implementation contexts and barriers, thus enhancing the effectiveness and sustainability of a tobacco-free workplace program.

\section{Contributions To The Literature:}

- This is the first study using mixed methods to adapt and evaluate the implementation of a comprehensive tobacco-free workplace program within behavioral health settings.

- We illustrate full application of the RE-AIM framework using mixed methods, yielding an understanding of contextual and multilevel (organizational, community, provider) factors influencing implementation, and explain results on each dimension.

- Study findings present an effective model in building capacity for integrating evidence-based practices for treating tobacco dependence via a formative evaluation process that facilitated program uptake by 
developing flexible, tailored and sustainable strategies responsive to the real-world conditions of diverse settings.

\section{Background}

Behavioral health clients (BHCs) (those with mental and substance use disorders) represent only $21 \%$ of the population, yet consume about half of the cigarettes sold in the United States [1] with smoking prevalence rates nearly five times that of the general population [2-7]. Consequently, these individuals account for $50 \%$ of annual smoking-related deaths [8]. From 2005-2016, smoking rates among the general population have declined steadily from $20.9 \%$ to $15.5 \%$ [9]. However, smoking rates for BHCs remain relatively static, and significantly higher, at $35.8 \%$ [9]. Practice guidelines $[2,10,11]$ recommend that smokers with and without behavioral challenges receive the same evidence-based-practices (EBPs) [12] proven effective in helping individuals in both groups to successfully quit smoking $[13,14]$ at the same rates $[15,16]$. Nonetheless, BHCs are less likely to quit smoking compared to those in the general population $[1,3]$ despite research showing equally high motivation to quit among both groups [17-19].

While many factors influence the persistently high smoking rates among BHCs, a significant contributor is the hesitancy of psychiatrists and behavioral health clinicians to offer specialized care for quitting [20,21]. In 2016 , only $48.9 \%$ of mental health facilities screened patients for tobacco use, only $37.6 \%$ of programs provided tobacco cessation counseling, and only $26.2 \%$ offered nicotine replacement therapy (NRT) [22]. The main barriers to implementing tobacco cessation services within behavioral health treatment centers (BHTCs) include several clinician-level factors such as lack of effective training on treating tobacco dependence [23, 24]; undervaluing of tobacco addiction as a problem [25]; lack of, or failure to, enforce tobacco-free workplace (TFW) policies [2]; and misconceptions regarding motivation for, and the behavioral effects of, quitting smoking for BHCs [12]. Clinicians also widely believe quitting smoking will jeopardize substance recovery efforts and trigger depression, aggressive behavior, suicide attempts or self-harming behaviors [20]. Although evidence indicates these clinician beliefs are myths [20], they persist and have the strongest negative impact [26,27], along with organizational barriers [28], on successful implementation of tobacco cessation interventions. Given the systemic nature of these barriers, organizational change is recommended $[29,30]$.

Taking Texas Tobacco Free (TTTF) is a multicomponent TFW program assisting Local Mental Health Authorities (LMHAs) throughout Texas to implement comprehensive and sustainable tobacco cessation [31], designed to target known implementation barriers across diverse levels of influence - organizational, community, clinician, and client. LMHAs are state agencies that administer and provide behavioral health services across Texas according to geographic region through non-profit community mental health centers [32]. As a comprehensive TFW program, TTTF includes a TFW policy, clinician training in evidence-based treatments, integration of tobacco use assessments (TUAs) into routine practice, staff education on hazards of tobacco use, community outreach, and assistance with NRT provision [2]. TTTF was effective in building capacity for the integration of EBPs in assessing and treating tobacco dependence and helping clients and employees within 18 LMHAs successfully quit smoking [33]. 
To facilitate understanding and overcoming of barriers, implementation researchers are increasingly adopting mixed methods designs [34]. Combining qualitative and quantitative approaches provides a more comprehensive understanding of implementation issues and greater knowledge yield than either method alone [35].

Although widely recommended in implementation research [34, 36-39], few studies have applied mixed methods in evaluating smoking cessation interventions. Interventional studies have focused on evaluating the use of mono, rather than multicomponent smoking cessation interventions, such as the effectiveness of tailored smoking cessation training for the elderly [40] and for youth [41], and of financial incentives on quitting [42]. One exception is a mixed methods evaluation of policy implementation of a national public health guideline for smoking cessation in the UK, focused on pre- and post-implementation changes. [43]

This study adopts a three-phase mixed methods design to examine the adaptation, dissemination and implementation of TTTF within two LMHAs overseeing 17 community clinics. The current study addresses limitations of prior research in implementation through using mixed methods to both adapt interventions and evaluate the processes and outcomes of implementing a comprehensive TFW program within BHTCs.

\section{Methods}

\section{Study design}

Following the successful implementation of TTTF within 18 LMHAs [31, 33], we were funded to enable its statewide dissemination through active and passive means and to implement a more cost-effective TTTF within additional LMHAs, this time using a mixed methods approach not included in the prior implementation. Researchers recommend the use of qualitative and mixed methods approaches to address the complexities involved in implementing interventions across diverse contexts [44] and for developing strategies that facilitate the implementation of EBPs $[34,36]$. Mixed methods is particularly suited to evaluating complex interventions. The quantitative component assesses program impact, while the qualitative provides insight into contextual factors and change processes that are key to program adaptation and implementation $[45,46]$, facilitating effective integration of EBPs into local contexts. Our purpose in using a mixed methods design was for, complementarity, (i.e. using quantitative data to examine outcomes and qualitative to examine processes), and expansion, (i.e. qualitative data is used to explain results of quantitative analyses) [47].

We used a multistage evaluation mixed methods design [48], consisting of three phases, each with a different aim and a different core mixed methods design (see Figure 1). Specifically: Phase 1) formative evaluation or needs assessment (pre-implementation) - to identify site-specific contextual factors that might affect implementation of TTTF to tailor the program to fit local contexts; Phase 2) program implementation - to assess the delivery and uptake of TTTF components within LMHAs; and Phase 3) summative evaluation (post-implementation) - to evaluate program outcomes and characterize processes influencing program reach, effectiveness, adoption, implementation, and maintenance [49]. RE-AIM was selected as a guiding framework as it was developed to translate behavioral interventions into sustainable practice [49]. Full application of the model requires in-depth understanding of contextual and multilevel 
factors influencing implementation, that use of both qualitative and quantitative methods provide, to understand and to explain reasons for outcomes on the RE-AIM dimensions [50]. Phases were interactive, with each phase building upon prior findings, and with iterative quantitative and qualitative data collection and analyses over the three phases [48]. The quantitative component had greater priority [48].

\section{Participants and setting}

This project has Institutional Review Board approval. Participation was open to any interested LMHAs in Texas who had not implemented the program previously. Our LMHA partner in the project, Integral Care, recruited centers through targeted mailings to 20 LMHA chief executive officers (CEOs) assessing interest. Three LMHAs indicated interest, provided consent for participation, and enrolled: one withdrew mid-course due to competing organizational priorities. This paper focuses on the two remaining LMHAs. LMHA1 was in a large urban area and comprised two community BHTCs annually serving 10,247 unique clients $(213,498$ contacts). LMHA2 was in a mixed urban and rural area, spanning 25,000 square miles, managed 15 community BHTCs and annually served 6,538 unique clients (339,158 contacts).

\section{Intervention: Taking Texas Tobacco Free}

Previously described in detail $[31,33,51,52]$, TTTF is a multicomponent, comprehensive, and sustainable TFW program that is implemented through an academic-community partnership. TTTF entails adoption of various EBPs in tobacco cessation [2, 10], including: 1) policy implementation and enforcement; 2) integration of TUAs into routine practice; 3 ) provision of tobacco cessation services (e.g., behavioral counseling, NRT); 4) specialized provider training on treating tobacco dependence, i.e., Motivational Interviewing (MI); and 5) and community outreach (Figure 2). Program components target challenges and misperceptions regarding tobacco dependence among $\mathrm{BHCs}$, encourage program buy-in and integration into local settings to reduce tobacco use and exposure to environmental tobacco smoke (ETS), and ultimately, prevent tobacco-related cancers and other diseases. TTTF was designed to increase organizational capacity for the provision of EBPs for tobacco use, because the delivery of such interventions has proven effective in increasing quit attempts and cessation among clients [10]. Therefore, TTTF's success is measured through RE-AIM targets as opposed to smoking cessation, reduced morbidity, etc. (cf. [53, 54]). We followed the Template for Intervention Description and Replication (TIDieR) Checklist [55] for describing interventions (Additional file 1).

\section{Data collection}

This study used a pre/post design with additional data collected during the active implementation phase to monitor program delivery and implementation. Data collection instruments used in this study are reported according to phase below, and described in Table 1. 
Table 1: Aims, methods, data collection and analysis according to phase

\section{Phase/Design/Timing}

Phase 1: Organizational

Preparation/ Preimplementation

\section{Formative Evaluation}

Aim: to identify LMHA traits, readiness to implement change and site-specific contextual factors to guide program development

Timing: Baseline - months 1-6, pre-implementation

\section{Type and description of data collection and analysis}

QUANTITATIVE Measures: descriptive analysis; All surveys are multiple choice

Organizational Readiness to Implement Change (ORIC). subscales, formed from 24 items:

-change valence efficacy toward change $\cdot$ change commitment $\bullet$ knowledge needed for change $\cdot$ resources

Demographics form: • annual client contacts $\cdot$ unique clients annually $\cdot$ full-time employees

Clinic leader survey: 27 items •current quitting tobacco services -attitudes re: TFW programs implementation challenges -tobacco prevalence among employees

Clinician survey: 27 items $\cdot k$ nowledge and training on tobacco use $\cdot$ current practice of EBPs to treat tobacco use

Employee survey - 25 items (staff, providers, leaders): •personal tobacco history $\cdot$ quit attempts $\cdot$ tobacco training

Knowledge test - 10 items (identical, within session type (clinician vs. general), given directly pre \& post receipt of tobacco education session): 'tobacco dependence treatments (clinicians) •hazards of tobacco use (general)

\section{QUALITATIVE Approaches: Thematic \& constant comparison} analysis

Participant observation - (4) initial meetings and site visits; (6) Program champion consultations

Staff focus groups (2; $N=23$ ): • unique clinic traits •views re: TFW programs implementation facilitators, barriers

\section{Phase 2: Program Implementation:}

Aim: to monitor, adapt and implement TTTF into LMHAs

*Timing: months 6-12, active implementation

\section{QUANTITATIVE Measures: Descriptive analysis}

Monthly TUA, NRT and community outreach logs: •TUAs conducted $\cdot$ NRT distributed $\cdot$ community events held

\section{QUALITATIVE Approaches: Thematic and constant comparison analysis}

Participant observation - (4) periodic site visits, and (6) Program champion consultations

Client focus groups $(1 ; \mathrm{N}=5)$ : • smoking history, current use •clinic quit services offered $\cdot$ cessation facilitators 
Staff focus groups $(2 ; \mathrm{N}=20)$ : $\cdot$ implementation challenges and facilitators $\cdot$ additional needs $\cdot$ program adaptations

Phase 3: Summative Evaluation:

Aim: evaluate program outcomes and explain processes influencing RE-AIM dimensions reach

*Timing: 2-6 months post

\section{QUANTITATIVE Measures: descriptive analysis}

Repeat of: Clinic leader survey; clinician survey; and all employee survey.

\section{QUALITATIVE Approaches: Thematic and constant comparison analysis}

Participant observation - (2) periodic site visits, and (4) program champion consultations

Staff focus groups $(2 ; \mathrm{N}=18)$ : $\cdot$ EBP integration effectiveness -implementation processes $\cdot$ improvements needed

*Phase 2 and 3 are not discrete, as program components began continued through summative evaluation. aShea CM, Jacobs SR, Esserman DA, Bruce K, Weiner BJ. Organizational readiness for implementing change: a psychometric assessment of a new measure. Implementation Science. 2014;9(1):7. LMHA = local mental health authority; TFW=tobacco-free workplace; TUA=tobacco use assessment; NRT=nicotine replacement therapy; EBP=evidence-based practice.

\section{PHASE 1: Formative evaluation}

The initial step in adopting TTTF was preparing for program implementation through developing each LMHAs' $100 \%$ TFW policy that included electronic nicotine delivery systems (ENDS), training clinicians, and champions, in treating tobacco dependence, educating staff about tobacco hazards, and integrating EBPs in tobacco cessation into clinical practice $[2,10]$, and tailoring materials to centers' needs. While TTTF team members consulted with LMHA leadership on policy drafting, we encouraged centers to confer with their members and community in developing their own TFW policy. Each LMHA designated one program champion to oversee program implementation and maintenance according to a recommended timeline [33], and facilitate and steward successful organizational change [56,57]. TTTF team members provided guidance and practical advice throughout program implementation.

Quantitative data included pre-implementation clinic leader, clinician and employee surveys, LMHA demographics, pre- and post-knowledge tests, and the Organizational Readiness to Implement Change (ORIC) [58] survey that assessed organizational characteristics and needs regarding knowledge, skills, practice, and readiness to implement change [scale of 1 (agree) - 5 (disagree), higher scores indicate greater commitment to change], (Table 1 describes instruments). Qualitative data included preimplementation staff focus groups, field visits and champion consultations. Analysis of quantitative data informed sample selection for the pre-implementation focus groups. We purposively selected a 
heterogeneous sample for the focus groups, selecting participants who had expressed apprehension of TTTF to hear and address their concerns.

\section{PHASE 2: Program implementation}

The active implementation phase entailed adoption of various EBPs in tobacco cessation $[2,10]$ as described in Figure 2. TUAs are an empirically based method to increase quit attempts [2], which in this program consisted of documenting current, and history of, tobacco use; prior quit attempts and methods used; NRT use; and clients' readiness to quit. Counselors facilitated smoking cessation groups using a validated and effective smoking curriculum, developed specifically for BHCs $[59,60]$. TTTF staff delivered an 8-hour training in MI to participating LMHAs, as guidelines indicate the most successful evidence-based interventions combine the delivery of behavioral and pharmacological support [10, 61]. In accordance with best practices, this combined therapy approach attends to clients' needs whether they are ready for smoking cessation treatments or motivational treatments to quit in the future $[10,25]$. Community outreach focused on expanding and sustaining community TFW programs to address high tobacco use rates among this population.

Quantitative data included LMHA quarterly reports submitted by champions, documenting TUAs administered and quantities and types of NRT distributed to staff and clients, and descriptions of monthly community outreach events. Various qualitative methods - mid-implementation staff, and separately, client, focus groups; site visits; and champion consultations - were used to engage program partners collaboratively, examine and understand implementation processes and challenges in greater depth, and make program adjustments as needed.

To facilitate documentation of client tobacco use, centers were asked to integrate TUAs into the electronic health record. Establishing use of these audit and monitoring methods are recognized as enhancing program success and sustainability [16]. Quarterly reports served two functions: (1) provided ongoing monitoring of program component delivery, allowing team members to intervene and suggest potential adjustments to these processes; and (2) provided data for summative evaluation.

\section{PHASE 3: Summative evaluation}

The summative evaluation phase consisted of LMHAs' ongoing implementation of evidence-based program components. Post-implementation, with the exception of the ORIC, we administered all surveys (center leader, clinician, and employee), continued monitoring implementation through quarterly TUA and NRT logs, and conducted staff focus groups, site visits and champion consultations. All qualitative methods focused on examining and understanding how and why results were obtained for program outcomes.

\section{Data Analysis}

\section{Quantitative}

Quantitative data were analyzed using SAS 9.4 [62] (Table 1). Descriptive statistics, including mean and standard deviation (SD) and percent, were provided for continuous and categorical variables of interest, 
respectively. The distribution of variables between pre- and post-implementation were examined using chisquare tests, as pre- and post- data were unmatched at the participant level. Alpha was set at 0.05.

\section{Qualitative}

Focus groups and interviews were conducted using interview guides (available upon request from corresponding author), transcribed verbatim and uploaded to Atlas.ti8 (v8.4) along with all other qualitative data to facilitate data management. Qualitative data were coded inductively from themes drawn from the data as well as deductively, according to the RE-AIM dimensions. Three team members trained in qualitative research independently coded initial transcripts to develop a coding frame. Coding discrepancies were discussed and reconciled until agreement was reached, refining codes until a final coding frame was agreed upon and reapplied to all transcripts [63]. Coding and analysis proceeded iteratively across each stage of data collection. Constant comparison of data - a continuous process of comparing emerging data within and across previously coded transcripts - was used to refine themes, avoid redundancy, ensure fittingness of themes, and check accurate accounting of the data set [64]. Analyst triangulation, where two or more analysts independently code the same data and compare findings, was used to ensure congruency and credibility of findings [65].

\section{Mixed methods integration}

Quantitative and qualitative data were analyzed separately, and merged at the end of each phase. Qualitative analysts remained blinded to quantitative results during qualitative data analysis, until data merging. Various types of integration were used to mix the quantitative and qualitative data, aligned with the different core mixed methods designs and aims of each study phase. In phase 1 (formative evaluation), the qualitative data was used to build and adapt intervention features to the local context. In phase 2 (program implementation and monitoring) qualitative and quantitative data were compared and connected to make ongoing program adjustments that facilitated successful implementation. In phase 3 (program evaluation) qualitative and quantitative data from phases $1-3$ were connected, that is, qualitative data on the implementation processes was used to explain the quantitative outcomes [66].

\section{Results}

\section{Phase 1}

Scores on the ORIC subscales for both LMHAs were as follows: commitment $=4.0(S D=.78)$; valence $=4.6$ $(\mathrm{SD}=.53)$; efficacy = $4.0(\mathrm{SD}=.86) ;$ knowledge = $3.5(\mathrm{SD}=1.16)$; resources = $3.7(\mathrm{SD}=0.94)$; and total $=4.0$ $(\mathrm{SD}=.73)$, showing a high degree of readiness to implement change. Regarding existing TFW policy, center leaders reported: 1 clinic (4.35\%) had a TFW policy that included e-cigarettes; 4 (17.39\%) regularly conducted TUAs and noted them within clinical records; 1 (4.35\%) routinely provided tobacco cessation services for clients wanting to quit; and $14(61 \%)$ had no existing TFW policy and did not provide assessments or any cessation services. However, regarding commitment to the TFW policy implementation at baseline, according to center leader surveys, $90 \%$ of employees ( $40 \%$ agree, $50 \%$ somewhat agree) were 
committed to implementing this policy change. Clinician survey results indicate only $25 \%$ of clinicians were providing TUAs pre-implementation.

Qualitative findings were used to adapt the program to individual centers through development of: 1) program materials, i.e., posters representing center clients regarding age, ethnicity and language; and 2) additional resources tailored to the needs of special populations, including pregnant women, women with infants and young children. Materials included educational brochures providing guidance and specific recommendations on EBPs for treating tobacco dependence within these specialized subpopulations. These intervention adaptations enhanced the delivery, reach, and implementation of TTTF.

Qualitative analysis showed that while the majority of LMHA staff were onboard with becoming tobaccofree, some, particularly smokers, were apprehensive regarding program implementation. In fact, many felt that staff apprehension was primary and greater than that of clients:

I anticipate a struggle with staff here who may be struggling with the new policy and then conveying that to consumers. 'You can do this [stop smoking] when I'm not sure I can do this'...I think the staff is going to be harder...I think our clients will follow what our staff does, for the most part, if we set a good example. (Program director, smoker, LMHA2)

Other concerns and apprehensions regarding program implementation included perceived violation of staff and clients' rights to smoke, and staff's expectations that client resistance would manifest as violent behavior, as well as attrition, due to becoming tobacco-free: "Our staff's concern would be behaviors from our consumers that we're going to try to prevent smoking. They're going to act out and then they're going to hurt us." (Nursing director, LMHA1). However, generally, staff expressed confidence that once implementation had started, staff and clients would accept the program: "They'll bicker about it first, but then everyone'll get on board." (Counselor, LMHA2)

\section{Phase 2}

TUA and NRT quarterly logs indicated implementation of each of these program initiatives. Both LMHAs were dispensing NRT to clients at about the same rates, $₫ 4$ boxes of patches monthly. Employees at LMHA2 used 2 packages of gum and 1 box of patches quarterly, while no employees at LMHA1 requested NRT. LMHA2 monthly logs show a slight decline in TUAs conducted during implementation (September: 812; October: 585; November: 483); however, LMHA1 saw an almost 26.42\% decline (September: 1200; October: 1690; November: 883) in TUAs conducted following the initial months of implementation. Qualitative findings explained this decline by revealing that prescribers from LMHA1 were conducting TUAs and were protesting that time constraints kept them from completing these assessments. At LMHA2, TUAs were conducted by clinicians and did not decrease considerably over time. Thus, triangulation of quantitative and qualitative methods [65] explained how this program delivery decision at LMHA1 hindered implementation of TUAs. "The prescribers do them [TUAs]...the issue we've had is doctors not having the time of really being able to go over them in depth." (Counselor, LMHA1). Once LMHA1 shifted the administering of TUAs to clinicians, numbers increased (December: 1446; January: 1764; February: 1615). 
Additionally, qualitative data revealed limitations to program delivery and implementation by LMHA. At LMHA1, clients and clinicians reported a need to tailor educational materials, which TTTF staff complied with, to fit clients' advanced readiness to change, and challenges of not offering after-hours smoking cessation groups. Implementation at LMHA2 was hindered initially by not including employees in NRT distribution along with clients, which was soon corrected. Furthermore, LMHA2 did not offer any smoking cessation groups. Results from the qualitative and quantitative data collection during implementation were connected to build and adjust program strategies and materials, address implementation challenges and understand processes to further enhance program implementation and sustainability.

\section{Phase 3}

Detailed results evaluating program implementation according to how each RE-AIM dimension was measured within this study, along with qualitative and mixed methods findings on each dimension, is included in Table 2. Analysis of qualitative data produced five themes guided by the RE-AIM framework: factors related to Reach, Effectiveness, Adoption, Implementation and Maintenance. (Table 3 provides quotes supporting each theme.)

Table 2. Quantitative, Qualitative and Mixed Methods Results on RE-AlM Dimensions 


\section{RE-AIM dimension \\ Quantitative Results}

Qualitative

Findings

Connecting Qualitative and Quantitative Findings

Reachmeasured via increases over baseline in:

1) percentage of clinicians trained in tobacco interventions;

2) percentage of employees trained in basic tobacco education;

3) number of community education and outreach events conducted and people reached

\section{Effectiveness-} assessed via:

1) establishment of enforced and comprehensive TFW policies in all LMHAs;

2) increases in the self-reported acceptability of TFW programs;

3) significant decreases over baseline in the proportion of LMHA employees using tobacco

\section{Significant increases over baseline:}

1) clinicians: $7 \%-77 \%$ to $22 \%-84 \%$; all p $<.001$ on: assessing client tobacco use, pharmacotherapy use, the effects of nicotine on psychiatric meds., use of counseling and behavioral therapies to treat tobacco use, hazards of smoking on BHCs;

2) employees: $34.6 \%$ to $88.2 \%$; $p<.001$;

3) 85754 people reached via community outreach-health fairs, social media and website through May 2018

\section{Theme: Factors related to Reach - Categories:}

1) Clinician and staff views on tobacco training

2) Community attitudes towards TFW programs
Qualitative findings support quantitative results:
1)Illustrating how clinicians and staff valued the tobacco training as essential to implementing change
2)Indicating acceptability of TWF within the greater community

\section{Significant changes over baseline:}

1) Both LMHAs successfully established a 100\% TFW policy; preimplementation only $4.17 \%$ of clinic leaders reported LMHA enforced a TFW policy providing quitting tobacco services to clients;

2) No significant changes in TFW program acceptability were seen pre/post, as employee motivation $(80 \%)$, determination $(85.71 \%)$, and confidence in ability to sustain a TFW (95.24\%) were high pre-implementation;

3) No significant decreases $(9.82 \%$ à $10.19 \%$ ) in number of employee smokers. However, average total quit attempts doubled, 4.89 to 10.42 postimplementation

\section{Theme: Factors related to Effectiveness- Categories:}

1)Questioning or supporting myths about TFW policy

2)Staff attitudes on TFW programs

3)Employee experiences of quitting smoking

\section{Qualitative findings from each LMHA differed compared to quantitative:}

1) LMHA2 enforced policy consistently, no employee or client policy violations reported; LMHA1 reported policy was inconsistently enforced due to fear of provoking client violence;

2) LHMA2 overcame TFW program misconceptions, LMHA1 did not;

3)LMHA2 reported one employee reported quitting, and two reducing smoking due to TTTF
Adoptionassessed via
Significant increases postimplementation over baseline:
Theme: Factors related to
Qualitative findings were applied to 
significant increases over baseline in:

1) the proportion of clinicians providing EBPs;

2) the number of TUAs conducted;

3) self-reported compliance with TFW policy consistent practices

\section{Implementation- assessed via self-reported program fidelity in:}
1) number of passive dissemination materials distributed;

2) number of championinitiated trainings;

3) self-reported consistency with step-bystep implementation guide components

\section{Maintenance-} establishment within LMHAs of systems to:

1) monitor and document TUA provision;

2) obtain and distribute NRT to clients and staff;

3) incorporate tobacco education into

1) Clinician provision of EBPs (chi square $=14.70, d f=1, p<.001$ ); specifically, behavioral counseling (chi square= 6.69, $d f=1, p=.010$ ); NRT (chi square $=31.98, \mathrm{df}=1, \mathrm{p}<.001$ ); but not for provision of non-nicotine medications (chi square=2.87, $\mathrm{df}=1, \mathrm{p}=.090$ ).

2) TUAs provided rose from $25 \%$ pre to $40 \%$ post (chi square $=9.99, \mathrm{df}=1$, $\mathrm{p}<.005)$;

3) $92 \%$ of employees reported TFW policy compliance

\section{Post-implementation, program fidelity regarding:}

1) passive dissemination materials 675 posters, 2650 rack cards;

2) LMHA2 CEO and champions from both LMHAs, presented their experiences on implementing TTTF at a national behavioral health conference with TTTF staff;

3) Each center successfully implemented the TFW program and all major program components
AdoptionCategories:

1) Contextual

factors

impacting

program uptake: facilitators and

barriers

2)Practices compliant with

TFW policy;

3)Clinician views

of tobacco

cessation

interventions facilitate and adapt uptake of TTTF:

1) Identified barriers and facilitators to

program

implementation;

2) Noted practicing of novel strategies to reduce client tobacco use;

3) Modified or adjusted delivery of program initiatives during active implementation phase

Theme: Factors related to ImplementationCategories:

1) Tailoring dissemination and implementation materials/ strategies

\section{2) Champion} initiative

3) Program implementation fidelity
Qualitative findings were applied to tailor TTTF to

LMHA specifics and note areas needing improvement:

1) Development of various site-specific program materials and strategies;

2) While champions effectively lead implementation efforts, neither

initiated center trainings

3) Variations between LMHAs in policy enforcement

\section{Systems established within LMHAs:}

1) To monitor and document TUAs, 13659 TUAs were delivered, 12377 unduplicated;

2) NRT distribution 89.5 boxes of patches, 10 boxes of gum, 41 boxes of lozenges to clients and employees;

3) $48 \%$ of clinic leaders reported implementing tobacco-free training into new employee orientation and $26 \%$ into annual training requirements
Theme: Factors related to Maintenance Categories:

1) Functional delivery systems

2) Attitudes about sustaining program initiatives

3) Integration into organizational culture
Qualitative findings were used to improve delivery and assess sustainability:

1)Delivery of TUAs was modified in LMHA1;

2) Clinicians valued TUAs and NRT as effective quit tools; NRT delivery was extended to employees also at LMHA2 
TFW = tobacco - free workplace; BHCs = behavioral health clients; LMHAs = local mental health authorities; EBP's = evidence-based practices; TTTF = Taking Texas Tobacco Free; TUAs = tobacco use assessments; NRT = nicotine replacement therapy; $\mathrm{CEO}=$ chief executive officer.

\section{Reach}

Reach focused on changes in clinician and staff training regarding tobacco, and community outreach. Significant outcomes were seen in clinician trainings in evidence-based tobacco cessation interventions and in educating staff on tobacco use hazards through cost-effective videoconference technology. Knowledge increase for clinicians pre- $(\mathrm{N}=286$, Mean 4.60) and post-education $(\mathrm{N}=268$, Mean 6.51) were significant $(p<.0001)$, and for general staff, pre- $(\mathrm{N}=153$, Mean 4.29), and post-education $(\mathrm{N}=108$, Mean 6.69, $p<.0001)$. Through outreach, we reached 973 professionals from participating LMHAs and various other community agencies via specialized clinical training and presentations on how to implement the TTTF program and become tobacco-free. Qualitatively, clinicians reported valuing the tobacco treatment training as vital to treating tobacco dependence and getting staff on board with program implementation. Likewise, general staff reported valuing the tobacco education training as helpful in understanding and assisting tobaccodependent clients. Clinicians also related that attitudes in their greater communities were supportive of tobacco-free environments, which facilitated TFW program implementation at LMHAs.

\section{Effectiveness}

Effectiveness focused on the establishment, enforcement and acceptability of TFW policies, and decreases over baseline of numbers of LMHA employees using tobacco. Quantitative results indicated full implementation of TFW policies in both participating LMHAs, leading them to become $100 \%$ TFWs, protecting thousands of employees, clients and visitors from ETS. Although participating LMHAs established TFW policies, staff focus group participants reported variations in policy enforcement in the two LMHAs. The CEO of LMHA2 reported that in enforcing the TFW policy, they realized their initial fears regarding policy implementation were unfounded misconceptions, which she repeatedly stressed to staff as a strong supporter and implementation TFW program leader (Table 3). As a result, LMHA2 enforced the policy consistently with clients and reported no violations among employees. Whereas during focus groups, LMHA1 staff repeatedly supported the misconception that enforcing TFW policies would precipitate client acting-out, and reported not enforcing the policy among clients due to fear of provoking violent behavior. Neither LMHA reported increases in client violent behavior, post-implementation. It is notable that these qualitative findings conflict with post-implementation survey data indicating adoption and enforcement of $100 \%$ TFW policies in both LMHAs. Additionally, LMHA2 employees were on board with enforcing their TFW policy and program, and reported policy integration into their workplace culture. LMHA1 staff reported that 
they should not be burdened with policy enforcement, signaling their reticence to accept and adopt the program fully. No employees reported quitting smoking at LMHA1; however, the percentage who smoked were few. At LMHA2, one employee reported quitting smoking because of TFW policy implementation during a focus group, and two others reported cutting down on smoking.

Table 3. Themes: factors related to the reach, effectiveness, adoption, implementation and maintenance of the TTTF program 


\section{Theme \& Categories}

\section{Factors related to Reach:}

\section{Categories:}

1) Clinician and staff views on training

2) Community attitudes towards TFW programs
Context

Clinicians' and staff's views on tobacco training, as well as community attitudes toward tobacco use were supportive of TTTF and positively impacted reach

\section{Participant Quotes}

1) The more education you guys provided us, the better. Like how it [nicotine] interacts with psychiatric medications...I had NO idea about that! I was really shocked at how much I didn't know! No one ever told us that! I was like, how could I NOT know this? So they're cutting their chances of getting into wellness with their mental health when they're smoking...it's all about education. (CEO, LMHA2)

1) The training we received was fantastic, and getting it to staff prior to the implementation date, I think it really brings people on board. When you have your center saying 'We're behind you, we're going to help you, provide you with the tools you need to help you quit smoking...I think it really shows the staff as individuals that, 'Okay, these guys really, truly care for me as something other than a warm body working.' (Program Director,LMHA2)

2) My experience of (city) is that it's a very health-conscious community...I think there's just a cultural shift here towards healthy living. A lot of people are into healthy eating, healthy living, so that makes it easier. (Intervention Director, LMHA 1))

\section{Factors related to Effectiveness}

\section{Categories:}

1) Questioning or supporting myths/ fears about TFW programs

2) Staff attitudes towards TFW program

3) Staff experiences of quitting
Attitudes towards Myths/Fears about TFW programs can aid or hinder program adoption

Staff acceptance of TTTF facilitated program success

TTTF helped employees to quit/ manage tobacco

1) But there were a lot of things that people predicted would happen that didn't happen. You know, that people would be smoking at respite or in the group homes. Most of the fears that we feared, we had no reason to fear. I mean it went so well. (CEO, LMHA2)

1) I'll be honest, I feel intimidated to go up to people to tell them to stop smoking, that's one of my fears of going up to someone who's already having a really bad day and then setting them off and having a crisis situation or an aggressive situation...l'm not going to risk myself to tell him to stop smoking, personally...I don't know anyone else who does. (Counselor, LMHA1)

2) I can't see where there would be any barriers coming forward to sustain it [tobacco-free program]... because everybody has bought into it, because it IS policy. It's part of (LMHA2) now and I believe that everybody has accepted that... This is our environment for (LMHA2) now. (Program Director, LMHA2))

3) Because we're going to a non-smoking campus I thought, well, I guess I got to quit smoking. Because, I mean, if I'm not going to smoke at work when I'm stressed out I might as well just quit. And it seemed like it just got too complicated. I've got to plan my smoke breaks off campus and go somewhere where I can smoke and that's just too complicated! I don't need to smoke that bad! (Counselor, LMHA2)

Factors related to Adoption:
Site-specific contextual factors can either hinder
1) The facilitator is going to be the staff and to a large degree the staff that smoke, and I know this is hard but... staff to client - 'I'm going through this too.' I don't smoke on the grounds either anymore. So the facilitator to the 
Categories:

1) Contextual

factors affecting

program uptake

(facilitators and

barriers)

2) TFW

compliance

3) Clinician views

of tobacco

cessation

interventions or aid program

uptake

Clinicians reported

using novel

practices

compliant with

TFW policy

Clinicians reported benefits of using tobacco cessation interventions largest degree is the staff. (Program Director, smoker, LMHA2)

1) The support staff aren't trained in mental health and so they really shouldn't be the ones who are telling them [not to smoke]...And so, they don't say something when they're out there [smoking] and we don't say something. And so, yeah, even though we want to be smoke free, I don't feel like it's being addressed or enforced. [Barrier] (Counselor, LMHA1)

2) Introducing mindfulness and those relaxed breathing skills...they really appreciated that and it's something they can take with them, and delaying the first cigarette too. A client told me, 'If I can delay that first one, just for one hour I'm not going to smoke, and then at the end of that hour, OK, just for another hour, I'm not going to smoke, then I can get to lunch [smoke-free].' (Counselor, LMHA1)

3) I think the best thing we've done so far is implementing the [tobacco] assessment...We're asking the question now. It's not just 'Are you smoking?' It's 'Do you want to quit?' We're also giving them the opportunity and providing that assistance to quit if they request it. (Program Manager, LMHA2)

\section{Factors related to Implementation}

\section{Categories:}

1) Tailoring material/strategies

2) Championinitiated trainings

3) Program fidelity (consistent with Implementation Guide (IG); website)
Program

materials/

strategies tailored

to staff

suggestions for

their center

Champion's work load, and confidence-level

limited implementing TTTF

Staff shared

faithfully

implementing

main TTTF

components and

use of TTTF's IG

and website
1) My consumers, I go to their homes because they are 0 through 3 [years old]. So, their families, some of them are smokers...if there is a pamphlet that we could give them the education about it [smoking] ...because the consumer is the baby who's in danger. So we need to offer assistance to the parents who are putting the baby in that environment. (Early childhood intervention specialist, LMHA2)

1) They [consumers] were really interested in how addiction effects the brain...and doing more group activities because the activities in the book are more individualized... what was most impactful to them was connecting with each other, having the common ground of being able to support each other. (Counselor, LMHA1)

2) I don't feel prepared to lead staff trainings...and don't really have the time to set them up and keep them going. (Champion, LMHA2)

3) We found it [website] really helpful...a lot of our stuff is based on Betty Hardwick and Dallas Metro Care. We looked a lot and picked out the positive messaging, especially for

our policy. (CEO, LMHA2)

3) There was some withdrawal symptoms on the website that we printed out that were really useful to give them to take with them...there is a column for withdrawal symptoms and then another column for coping skills. And so, you're going to be angry, you're going to be depressed, and you might be irritable and here are some skills that you can use for those. Counselor, LMHAT)

\section{Factors related to Maintenance}

Enabling uptake via

adjustments to key initiatives
1) The prescribers do them [TUAs]. Every time they [clients] see a prescriber, they do an assessment with them...the issue we've had is the doctors, and not having the time of really being able to go over it more in depth. (Counselor, LMHA 1)

Page $17 / 30$ 


\begin{tabular}{|c|c|c|}
\hline \multirow{2}{*}{$\begin{array}{l}\text { Categories: } \\
\text { 1) Functional } \\
\text { delivery systems }\end{array}$} & delivery & $\begin{array}{l}\text { 2) The only thing I can foresee from a management point is } \\
\text { iust as we continue to arow and change we're going to have }\end{array}$ \\
\hline & $\begin{array}{l}\text { While aware of } \\
\text { challenges of } \\
\text { sustaining TFW } \\
\text { proaram staff }\end{array}$ & $\begin{array}{l}\text { to be mindful not to forget and let it [TTTF] drop. Like } \\
\text { already [5 months after implementation] we're seeing this } \\
\text { dwindling...So I think we'll have to be continuously proactive } \\
\text { on how we're going to, as the fire dies, ignite it over and over } \\
\text { again so that we keep this awareness fresh and keep these } \\
\text { s.rvices available (Anonvm }\end{array}$ \\
\hline $\begin{array}{l}\text { 2) Attitudes } \\
\text { towards } \\
\text { sustaining } \\
\text { program initiatives }\end{array}$ & & \\
\hline $\begin{array}{l}\text { sustaining } \\
\text { program initiatives }\end{array}$ & $\begin{array}{l}\text { To varying } \\
\text { degrees, changes } \\
\text { were seen in }\end{array}$ & $\begin{array}{l}\text { 2) When you're interacting with a patient, instead of just } \\
\text { saying 'Do you smoke? Yes, no, how much?' You go on to } \\
\text { say 'Do you want some assistance quitting?' And we } \\
\text { weren't doing that. We were just 'Do you smoke?' Check, } \\
\text { check...And so we never knew whether they wanted help to } \\
\text { quit or not...You got to take it to the next step....that is so } \\
\text { important. (CEO, LMHA2) }\end{array}$ \\
\hline & $\begin{array}{l}\text { culture in } \\
\text { participating } \\
\text { LMHAs }\end{array}$ & $\begin{array}{l}\text { 3) It's a change in the culture and I think that's the whole } \\
\text { point of the program is to change the culture and how we } \\
\text { see things and what the new normal is. Smoking has been } \\
\text { the normal and this process is changing all of that, it's just } \\
\text { going to take us a few months to get there. (Program } \\
\text { Manager, LMHA2) }\end{array}$ \\
\hline
\end{tabular}

TFW = tobacco - free workplace; TTTF = Taking Texas Tobacco Free; LMHAs = local mental health authorities; CEO = chief executive officer; TUAs = tobacco use assessments; IG = Implementation Guide.

\section{Adoption}

Increases in adoption measures (TUAs conducted, EBPs provided, TFW policy compliance), were significant, with clinicians trained and conducting 13,659 (12,377 unduplicated) TUAs during implementation. Diverse NRT products ( 140.5 boxes) were delivered and distributed to clients and employees, which indicates people were making quit attempts. Qualitatively, staff attitudes towards enforcing the TFW policy served as both an implementation barrier and a facilitator. In LMHA1, staff apprehensions served as a barrier to full program implementation while at LMHA2, staff program support facilitated becoming tobacco-free. Clinicians at both LMHAs were enthusiastic about providing novel EBPs for tobacco cessation to clients, such as delaying gratification and reported their effectiveness in helping clients manage smoking. Likewise, clients reported that the smoking cessation groups had helped them to quit smoking: "And l've got these mints [NRT] which, you know, they really, really work...But without the group support, I wouldn't have been able to do it." (Sandy, Client, LMHA1) Within one smoking cessation group at LMHA1, 2 out of the 3 participants $(66.66 \%)$ reported quitting smoking. Counselors of other groups reported that many clients had reduced their smoking significantly. Clinicians reported compliance with implementing practices consistent with upholding the TFW policy such as the TUAs, described as a vital step in helping clients in quitting smoking.

\section{Implementation}

Regarding implementation, quantitative assessment of program fidelity indicated each LMHA had successfully implemented all major TTTF components as intended. Qualitative implementation factors 
focused on staff and clients' suggested alterations to increase program fit to their center, and implementation consistency with the Implementation Guide (IG). Employees' suggestions led to the creation of various dissemination materials targeting their clients, including posters in Vietnamese, of young people and teens, and smokeless tobacco users, and information cards addressing the harms of ETS, especially on young children, and the hazards of smoking while pregnant. Employees reported consulting the website (www.takingtexastobaccofree.org) and the step-by-step IG and their usefulness in steering implementation efforts. Clients suggested adjustments to the smoking group curriculum, to reflect individuals in the preparation or action stage of change [67], rather than pre-contemplation: "It's [curriculum] geared towards smokers who haven't made the decision to stop smoking...but we have already made the decision to quit and that's why we signed up for the group" (Rick, Client, LMHA1)." Clients also asked for more group, rather than individual, smoking cessation materials and requested information on the neurobiology of nicotine addiction. We added these resources to the project website to address requests and improve implementation.

\section{Maintenance}

Regarding program maintenance, systems for monitoring and documenting TUA provision and NRT distribution were established, and modified where needed, to operate efficiently within each LMHA, and some tobacco education for new employees had been incorporated into annual trainings. Qualitative data indicate employees' general attitudes towards ongoing program maintenance between the two LMHAs varied. At LMHA1, the tobacco-free program was seen as having been implemented and then forgotten about; a project requiring periodic reviving, rather than being integrated into center culture. At LMHA2, employees reported integration of the program and program maintenance into the center to become part of organizational culture. Both LMHAs valued NRT as essential to helping their clients manage or quit smoking.

Areas in which program implementation were weak included: 1) lack of significant decrease over baseline of the number of employees using tobacco, as the distribution of smokers did not change significantly from pre- to post-implementation ( $x^{2}=6.79, \mathrm{df}=3, p=0.079$; and 2 ) lack of sustainability initiatives, i.e. neither LMHA provided champion-initiated trainings, or continued smoking cessation groups. Challenges reported by champions to providing in-house trainings included competing organizational duties and lacking confidence to lead such trainings.

\section{Discussion}

Study findings demonstrate that while implementation of program components was differential within and between LMHAs, TTTF's implementation was largely successful. Both LMHAs integrated tobacco cessation interventions and TUAs into routine practice, delivered evidence-based tobacco cessation interventions, recorded significant increases in clinician knowledge on how to address tobacco dependence, and dispensed NRT to clients. Although both LMHAs reported adopting a 100\% TFW policy, comparison of quantitative and qualitative data indicate inconsistent policy implementation and enforcement between the two agencies. While LMHA2 adopted and enforced a 100\% TFW policy, qualitative findings from staff focus 
groups indicated LMHA1 staff inconsistently enforced the policy out of fear of provoking violent behavior from clients. Policy adoption without policy enforcement is limited.

As researchers have noted, clinician misconceptions regarding treating tobacco dependence within BHTCs serve as the strongest organizational barrier to successful implementation of tobacco cessation programs [26-28]. In applying what they learned in the TTTF trainings on treating tobacco dependence among BHCs, LMHA2 staff overcame their initial misconceptions and fears regarding addressing smoking among their clients to faithfully integrate TTTF into their organization. The overcoming of this fundamental barrier served as a catalyst allowing LMHA2 staff and leadership to incorporate effective clinician training on EBP's to treat tobacco dependence, value tobacco addiction as a serious problem, and establish and enforce tobacco-free policies - thus addressing the most commonly cited implementation barriers to tobacco cessation programs within BHTCs $[2,12,23-25,32]$. Whereas at LMHA1, staff attitudes and misapprehensions regarding smoking and their BHCs persisted without change, resulting in partial program adoption due to lack of policy enforcement. Although TTTF team members continually attempted to correct these misconceptions by providing research evidence and prior program experience to the contrary, these erroneous beliefs continued unabated among LMHA1 staff. Qualitative data indicate the difference between attitudes at each LMHA towards TFW policy enforcement was largely influenced by program support, or lack thereof, of center leaders. The CEO of LMHA2 actively championed and remained abreast of program implementation, while LMHA1 center leadership adopted a hands-off approach, delegating implementation exclusively to managerial staff. Our findings are consistent with various studies citing the critical importance of support and direction from organizational leaders to successful program implementation [6870].

Qualitative data from clinicians and clients at LMHA1 indicate greater success in clients quitting smoking than at LMHA2. Differences in client quit rates at each LMHA may likely be attributed to the provision, or lack, of smoking cessation groups, which have proven effective in helping BHC's to quit as part of comprehensive tobacco-free programs $[25,59,60]$. Although LMHA 1 only held one series of smoking cessation groups, clinicians and clients reported that in one group alone, two out of the three group members $(66.66 \%)$ quit smoking with support from peers and NRT. LMHA2 did not provide any smoking cessation groups. At LMHA1, clinicians were enthusiastic, engaged and supportive in assisting clients in the smoking cessation groups, and clients attributed their success in quitting to the support they received from them. While staff at both LMHAs valued the provision of smoking cessation groups, their main obstacle was organizational, as neither operated in the evening or offered after-hours services, when clients were available for evening sessions.

Study findings on each of our individual program components is consistent with prior research supporting the effectiveness of TFW policies in reducing tobacco use [71, 72]; TUAs in increasing quit attempts [2]; EBPs in treating tobacco dependence [10,73]; and increasing cessation services provided by training clinicians on treating tobacco dependence $[24,74]$. Moreover, as a comprehensive organization-wide TFW program, TTTF's multicomponent model proved effective in addressing the many challenges influencing successful program implementation $[25,30]$ and affecting organizational change. Implementation scientists have stressed the importance of differentiating between core intervention components, and core

Page 20/30 
implementation components, i.e., the core implementation drivers required to implement intervention components, such as coaching or training [75].

Employing a mixed methods design allowed key program stakeholders to participate collaboratively via qualitative methods to shape core intervention components to their needs, and researchers to understand context-specific implementation facilitators, barriers and processes at individual LMHAs. Improving systems, organizational and community fit of intervention components enhanced core implementation components and addressed implementation challenges more effectively. Use of mixed methods also facilitated implementation by providing information on which intervention components were successfully adopted (or not), by whom, and why (or why not), expanding our understanding of what core implementation components need further development.

Identified areas of weakness in program implementation on the RE-AIM dimensions included: 1) absence of champion-initiated trainings (implementation); 2) inability to provide routine smoking cessation groups (adoption); and 3) continuing tobacco education - i.e., challenges integrating tobacco education into new employee orientation and training (maintenance). Although these components are on the clinician, systems, and organizational levels, the organization primarily determines them, as ultimately, they are due to prioritization of resources.

While champions' stewardship was vital in overseeing and organizing program implementation and maintenance efforts, neither took the next step to initiate any trainings in their LMHAs. Other studies support the importance of champions in leading successful implementation [76]. Champions reported competing duties and demands on their time and uncertainty in their ability to lead such trainings, as the main obstacles to providing in-house trainings. As both program champions reported similar challenges to providing and leading continuing tobacco education efforts, this indicates an area of needed improvement to the TTTF program, i.e., greater support and resources, such as train-the-trainer courses, to meet this implementation goal to ensure sustainability. Our future implementation programs will seek to address how to provide champions with the additional resources needed to transition from being managers to leaders of organizational change.

There are several limitations to this study. Some focus group participants may have had a stake in overreporting program success. To diminish the potential for social desirability bias in the qualitative data, we intentionally included program sympathizers and detractors to get a more accurate picture of implementation, inquired about - and encouraged participants to share - negative and positive experiences, and used varied qualitative data collection methods, triangulating across data sources to ensure rigor [65, 77]. Given the central role of context to successful implementation and the attention placed on this factor within this study, study findings are not necessarily applicable to other settings and populations. Our aim however, was not generalizability of findings; rather on the contrary, we sought to demonstrate and describe how we identified and responded to the needs of individual clinics to enhance program fit and implementation. Limitations for quantitative data included that not all surveyed stakeholders participated in data collection, despite solicitation, and inability to match pre-and post-data at participant-level. Also, as 
previously mentioned, client-level quantitative data on quit attempts among clients was not collected for comparison to qualitative reports.

\section{Conclusions}

Implementation of TTTF at both LMHAs increased organizational capacity in the provision of EBPs to treat tobacco use and dependence through successfully meeting the majority of our RE-AIM targets. Adopting a mixed methods approach enhanced TTTF program implementation, which allowed us to conduct a formative evaluation process to adapt implementation strategies to local contexts; evaluate program outcomes; and characterize processes influencing program implementation in two LMHAs (17 clinics). Mixing methods also involved program adopters and recipients as collaborators who directly influenced implementation by shaping core interventions to their individual context and needs, facilitating uptake. The collaboration of such key stakeholders was vital to enhancing program buy-in, adapting delivery systems, program content and materials, and ensuring maintenance; and alerted us to needed improvements in core implementation components. Study findings suggest that successful implementation of multilevel, evidence-based tobacco interventions requires an in-depth understanding of the implementation culture at the level of the clinician, client, organization, and community level, to better address barriers and support facilitators.

Study findings contribute to the development of flexible strategies and tailored interventions responsive to real-world conditions in diverse settings, which are better equipped to address implementation barriers. The need to address tobacco dependence among BHCs is imperative. This evaluation of the TTTF program presents a successful model for the implementation of an effective and sustainable evidence-based, TFW program in community organizations interested in becoming tobacco-free.

\section{Declarations}

\section{Availability of data and materials}

The datasets supporting the findings of this study are available, on reasonable request, from the corresponding author, Dr. Lorraine Reitzel at: Lrreitzel@uh.edu. Program materials are available online at our project website: www.takingtexastobaccofree.com.

\section{Acknowledgements}

The authors would like to acknowledge Hiroe Okamoto, MPH, and Sonakshee Shree, MPH, for their assistance with data analysis. We are grateful to the many clients, clinicians, staff and clinic leaders who generously shared their time and views with us to make this study possible.

\section{Funding}

Funding for this research was provided by the Cancer Prevention and Research Institute (PP160081; PI Reitzel) and the University of Houston (to Reitzel) from $07 / 31 / 16$ to $05 / 30 / 18$. The construction of this 
manuscript was supported by funding provided by the Cancer Prevention and Research Institute (PP170070; PI Reitzel; 07/31/17-08/30/20).

\section{Author's Contributions}

IML conceived of the study, collected, oversaw and contributed to qualitative data analysis, and wrote the manuscript. BK managed the project and collected quantitative data. TAC conducted quantitative data analysis, under the direction and assistance of DPO, and both edited the manuscript. KL assisted with quantitative data management. VCF edited and provided direction for writing up the manuscript, and along with BK, conducted staff and clinicians trainings. BK oversaw program implementation, and VCF and TW contributed to program implementation. LRR obtained funding to support this project, served as project director along with WTW, and lead the design and implementation of the program, and contributed to writing the manuscript. All authors read and approved the final manuscript.

\section{Ethics Declarations}

\section{Ethics approval and consent to participate}

This study was approved by the Institutional Review Board of the University of Houston (Study: 16541-EX (8506), approved, 07/13/2016; additional modifications approved 08/19/16). All participants provided oral or written consents.

\section{Consent for publication}

Not applicable.

\section{Competing interests}

The authors have no competing interests to declare.

\section{Abbreviations}

BHTCs: Behavioral health treatment centers

TTTF: Taking Texas Tobacco Free

NRT: Nicotine replacement therapy

RE-AIM: Reach, effectiveness, adoption, implementation, maintenance

BHCs: Behavioral health conditions

EBPs: Evidence-based practices

TFW: Tobacco-free workplace

LMHAs: Local mental health authorities 
TUA: Tobacco-use assessments

CEOs: Chief executive officers

ETS: Environmental tobacco smoke

Ml: Motivational interviewing

ORIC: Organizational Readiness to Implement Change

\section{References}

1. Lasser K, Boyd JW, Woolhandler S, Himmelstein DU, McCormick D, Bor DH. Smoking and mental illness: a population-based prevalence study. Jama. 2000;284(20):2606-10.

2. Best Practices for Comprehensive Tobacco Control Programs. [Internet]. US Department of Health and Human Services, Centers for Disease Control and Preventio, National Centers for Chronic Disease Prevention and Health Promotion, Office on Smoking and Health. 2014 [cited 15 Februrary 2018]. Available from: https://www.cdc.gov/tobacco/stateandcommunity/best_practices/pdfs/2014/comprehensive.pdf.

3. Current Smoking Among Adults-United States, 2005-2015 [Internet]. US Department of Health and Human Sciences, Centers for Disease Control and Prevention, National Centers for Chronic Disease Prevention and Health Promotion, Office on Smoking and Health. 2016 [cited April 24, 2018]. Available from: https://www.cdc.gov/mmwr/volums/65/wr/mm6544a2.htm.

4. Guydish J, Passalacqua E, Tajima B, Chan M, Chun J, Bostrom A. Smoking prevalence in addiction treatment: a review. Nicotine \& Tobacco Research. 2011:ntr048.

5. Knudsen HK. Implementation of smoking cessation treatment in substance use disorder treatment settings: a review. The American Journal of Drug and Alcohol Abuse. 2016:1-11.

6. Williams JM, Ziedonis D. Addressing tobacco among individuals with a mental illness or an addiction. Addictive behaviors. 2004;29(6):1067-83.

7. McKelvey K, Thrul J, Ramo D. Impact of quitting smoking and smoking cessation treatment on substance use outcomes: An updated and narrative review. Addictive behaviors. 2017;65:161-70.

8. Annamalai A, Singh N, O’Malley SS. Focus: Addiction: Smoking Use and Cessation Among People with Serious Mental Illness. The Yale journal of biology and medicine. 2015;88(3):271.

9. Jamal A, Phillips E, Gentzke AS, Homa DM, Babb SD, King BA, et al. Current cigarette smoking among adults-United States, 2016. Morbidity and Mortality Weekly Report. 2018;67(2):53.

10. Fiore M, Jaén, C. R., Baker, T. B., Bailey, W. C., Bennett, G., Benowitz, N. L et al. A clinical practice guideline for treating tobacco use and dependence: 2008 update: a US public health service report. American journal of preventive medicine. 2008;35(2):158.

11. Association AP. American Psychiatric Association Practice Guidelines for the Treatment of Patients with Substance Use Disorders2006 October 3, 2018:[71-89 pp.] [Cited 24 April 2018]. Available from: https://psychiatryonline.org/pb/assets/raw/sitewide/practice_guidelines/guidelines/substanceuse.pdf. 
12. Himelhoch S, Riddle J, Goldman HH. Barriers to implementing evidence-based smoking cessation practices in nine community mental health sites. Psychiatric Services. 2014;65(1):75-80.

13. Anthenelli RM, Benowitz NL, West R, St Aubin L, McRae T, Lawrence D, et al. Neuropsychiatric safety and efficacy of varenicline, bupropion, and nicotine patch in smokers with and without psychiatric disorders (EAGLES): a double-blind, randomised, placebo-controlled clinical trial. The Lancet. 2016;387(10037):2507-20.

14. Hitsman B, Moss TG, Montoya ID, George TP. Treatment of tobacco dependence in mental health and addictive disorders. The Canadian Journal of Psychiatry. 2009;54(6):368-78.

15. Meernik C, McCullough A, Ranney L, Walsh B, Goldstein AO. Evaluation of Community-Based Cessation Programs: How Do Smokers with Behavioral Health Conditions Fare? Community mental health journal. 2018;54(2):158-65.

16. Lawn S, Campion J. Achieving smoke-free mental health services: lessons from the past decade of implementation research. International journal of environmental research and public health. 2013;10(9):4224-44.

17. Kotz D, Brown J, West R. Predictive validity of the Motivation To Stop Scale (MTSS): a single-item measure of motivation to stop smoking. Drug and Alcohol Dependence. 2013;128(1):15-9.

18. Siru R, Hulse GK, Tait RJ. Assessing motivation to quit smoking in people with mental illness: a review. Addiction. 2009;104(5):719-33.

19. Stockings E, Bowman J, McElwaine K, Baker A, Terry M, Clancy R, et al. Readiness to quit smoking and quit attempts among Australian mental health inpatients. nicotine \& tobacco research. 2012;15(5):9429.

20. Prochaska JJ. Failure to treat tobacco use in mental health and addiction treatment settings: a form of harm reduction? Drug and alcohol dependence. 2010;110(3):177-82.

21. Prochaska JJ, Das S, Young-Wolff KC. Smoking, mental illness, and public health. Annual review of public health. 2017;38:165-85.

22. Marynak K, VanFrank B, Tetlow S, Mahoney M, Phillips E, Jamal A, et al. Tobacco cessation interventions and smoke-free policies in mental health and substance abuse treatment facilities-United States, 2016. Morbidity and Mortality Weekly Report. 2018;67(18):519.

23. Steinberg MB, Alvarez MS, Delnevo CD, Kaufman I, Cantor JC. Disparity of physicians' utilization of tobacco treatment services. American journal of health behavior. 2006;30(4):375-86.

24. Graydon MM, Corno CM, Schacht RL, Knoblach DJ, Wiprovnick AE, Thrash ST, et al. A statewide initiative to train behavioral health providers in smoking cessation. Translational behavioral medicine. 2018.

25. Williams JM, Zimmermann MH, Steinberg ML, Gandhi KK, Delnevo C, Steinberg MB, et al. A comprehensive model for mental health tobacco recovery in New Jersey. Administration and Policy in Mental Health and Mental Health Services Research. 2011;38(5):368-83.

26. Brown CH, Medoff D, Dickerson FB, Fang LJ, Lucksted A, Goldberg RW, et al. Factors influencing implementation of smoking cessation treatment within community mental health centers. Journal of dual diagnosis. 2015;11(2):145-50. 
27. Koch JR, Breland A. Behavioral healthcare staff attitudes and practices regarding consumer tobacco cessation services. The journal of behavioral health services \& research. 2017;44(3):399-413.

28. Knudsen HK, Studts JL, Boyd S, Roman PM. Structural and cultural barriers to the adoption of smoking cessation services in addiction treatment organizations. Journal of addictive diseases. 2010;29(3):294305.

29. Campbell BK, Wander N, Stark MJ, Holbert T. Treating cigarette smoking in drug-abusing clients. Journal of Substance Abuse Treatment. 1995;12(2):89-94.

30. Guydish J, Ziedonis D, Tajima B, Seward G, Passalacqua E, Chan M, et al. Addressing Tobacco Through Organizational Change (ATTOC) in residential addiction treatment settings. Drug and Alcohol Dependence. 2012;121(1-2):30-7.

31. Samaha HL, Correa-Fernández V, Lam C, Wilson WT, Kyburz B, Stacey T, et al. Addressing tobacco use among consumers and staff at behavioral health treatment facilities through comprehensive workplace programming. Health promotion practice. 2017;18(4):561-70.

32. Texas Department of State Health Services. Local Mental Health Authorities (LMHAs). [Available from: https://www.dshs.state.tx.us/mhsa/lmha-list/.

33. Correa-Fernández V, Wilson WT, Kyburz B, O'Connor DP, Stacey T, Williams T, et al. Evaluation of the Taking Texas Tobacco Free Workplace Program within behavioral health centers. Translational behavioral medicine. 2019;9(2):319-27.

34. Palinkas LA, Aarons GA, Horwitz S, Chamberlain P, Hurlburt M, Landsverk J. Mixed method designs in implementation research. Administration and Policy in Mental Health and Mental Health Services Research. 2011;38(1):44-53.

35. O'Cathain A, Murphy E, Nicholl J. Integration and publications as indicators of "yield" from mixed methods studies. Journal of Mixed Methods Research. 2007;1(2):147-63.

36. Proctor EK, Landsverk J, Aarons G, Chambers D, Glisson C, Mittman B. Implementation research in mental health services: an emerging science with conceptual, methodological, and training challenges. Administration and Policy in Mental Health and Mental Health Services Research. 2009;36(1):24-34.

37. Peters DH, Adam T, Alonge O, Agyepong IA, Tran N. Implementation research: what it is and how to do it. Bmj. 2013;347:f6753.

38. Peters DH, Tran NT, Adam T. Implementation research in health: a practical guide: World Health Organization; 2013.

39. Aarons GA, Fettes DL, Sommerfeld DH, Palinkas LA. Mixed methods for implementation research: application to evidence-based practice implementation and staff turnover in community-based organizations providing child welfare services. Child maltreatment. 2012;17(1):67-79.

40. Kerr S, Whyte R, Watson H, Tolson D, McFadyen AK. A mixed-methods evaluation of the effectiveness of tailored smoking cessation training for healthcare practitioners who work with older people. Worldviews on Evidence-Based Nursing. 2011;8(3):177-86.

41. Cain JJ, Dickinson WP, Fernald D, Bublitz C, Dickinson LM, West D. Family physicians and youth tobacco-free education: outcomes of the Colorado Tar Wars program. The Journal of the American Board of Family Medicine. 2006;19(6):579-89. 
42. Kim A, Kamyab K, Zhu J, Volpp K. Why are financial incentives not effective at influencing some smokers to quit? Results of a process evaluation of a worksite trial assessing the efficacy of financial incentives for smoking cessation. Journal of occupational and environmental medicine. 2011;53(1):627.

43. Huddlestone L, Sohal H, Paul C, Ratschen E. Complete smokefree policies in mental health inpatient settings: results from a mixed-methods evaluation before and after implementing national guidance. BMC health services research. 2018;18(1):542.

44. Southam-Gerow MA, Dorsey S. Qualitative and mixed methods research in dissemination and implementation science: introduction to the special issue. Journal of Clinical Child \& Adolescent Psychology. 2014;43(6):845-50.

45. Craig P, Dieppe P, Macintyre S, Michie S, Nazareth I, Petticrew M. Developing and evaluating complex interventions: the new Medical Research Council guidance. Bmj. 2008;337:a1655.

46. Mason J. Mixing methods in a qualitatively driven way. Qualitative research. 2006;6(1):9-25.

47. Greene JC, Caracelli VJ, Graham WF. Toward a conceptual framework for mixed-method evaluation designs. Educational evaluation and policy analysis. 1989;11(3):255-74.

48. Creswell JW, Clark VLP. Designing and conducting mixed methods research: Sage publications; 2017.

49. Glasgow RE, Vogt TM, Boles SM. Evaluating the public health impact of health promotion interventions: the RE-AIM framework. American journal of public health. 1999;89(9):1322-7.

50. Holtrop JS, Rabin BA, Glasgow RE. Qualitative approaches to use of the RE-AIM framework: rationale and methods. BMC health services research. 2018;18(1):177.

51. Correa-Fernández V, Wilson WT, Shedrick DA, Kyburz B, Samaha HL, Stacey T, et al. Implementation of a tobacco-free workplace program at a local mental health authority. Translational behavioral medicine. 2017;7(2):204-11.

52. Garey L NC, Martinez Leal I, Lam C, Wilson W T, Kyburz B, Stacey T, Correa-Fernández V, Williams T, Zvolensky M J, Reitzel L R. Organizational Moderators of Change in Behavioral Health Center Clinician and Staff Tobacco-related Knowledge Following Brief Education During a Comprehensive Tobacco-Free Workplace Program Implementation Currently under review.

53. Vidrine JI, Shete S, Cao Y, Greisinger A, Harmonson P, Sharp B, et al. Ask-Advise-Connect: a new approach to smoking treatment delivery in health care settings. JAMA internal medicine. 2013;173(6):458-64.

54. Vidrine JI, Shete S, Li Y, Cao Y, Alford MH, Galindo-Talton RM, et al. The ask-advise-connect approach for smokers in a safety net healthcare system: A group-randomized trial. American journal of preventive medicine. 2013;45(6):737-41.

55. Hoffmann TC, Glasziou PP, Boutron I, Milne R, Perera R, Moher D, et al. Better reporting of interventions: template for intervention description and replication (TIDieR) checklist and guide. Bmj. 2014;348:g1687.

56. Kirchner JE, Parker LE, Bonner LM, Fickel JJ, Yano EM, Ritchie MJ. Roles of managers, frontline staff and local champions, in implementing quality improvement: stakeholders' perspectives. Journal of Evaluation in Clinical Practice. 2012;18(1):63-9. 
57. Ziedonis DM, Zammarelli L, Seward G, Oliver K, Guydish J, Hobart M, et al. Addressing tobacco use through organizational change: a case study of an addiction treatment organization. Journal of Psychoactive Drugs. 2007;39(4):451-9.

58. Shea CM, Jacobs SR, Esserman DA, Bruce K, Weiner BJ. Organizational readiness for implementing change: a psychometric assessment of a new measure. Implementation Science. 2014;9(1):7.

59. Lee JG, Ranney LM, Goldstein AO, McCullough A, Fulton-Smith SM, Collins NO. Successful implementation of a wellness and tobacco cessation curriculum in psychosocial rehabilitation clubhouses. BMC Public Health. 2011;11(1):702.

60. Williams JM, Ziedonis DM, Vreeland B, Speelman-Edwards N, Zechner MR, Williams MT, et al. A wellness approach to addressing tobacco in mental health settings: Learning about healthy living. American Journal of Psychiatric Rehabilitation. 2009;12(4):352-69.

61. Hall SM, Prochaska JJ. Treatment of smokers with co-occurring disorders: emphasis on integration in mental health and addiction treatment settings. Annual Review of Clinical Psychology. 2009;5:409-31.

62. SAS Institute Inc. SAS (Version 9.4) [Computer Software]. Cary NSI, Inc.

63. Mayan MJ. Essentials of qualitative inquiry: Routledge; 2016.

64. Morse JM, Field P-A. Qualitative research methods for health professionals. 1995.

65. Patton MQ. Enhancing the quality and credibility of qualitative analysis. Health services research. 1999;34(5 Pt 2):1189.

66. Creswell JW. A concise introduction to mixed methods research: Sage Publications; 2014.

67. Prochaska JO, DiClemente CC. The transtheoretical approach. In: Norcross JCG, Marvin R. (eds.) editor. Handbook of psychotherapy integration. Oxford series in clinical psychology (2nd ed.). Oxford; New York: Oxford University Press; 2005. p. 147-71.

68. Crump CE, Earp JAL, Kozma CM, Hertz-Picciotto I. Effect of organization-level variables on differential employee participation in 10 federal worksite health promotion programs. Health Education Quarterly. 1996;23(2):204-23.

69. Emmons KM, Thompson B, McLerran D, Sorensen G, Linnan L, Basen-Engquist K, et al. The relationship between organizational characteristics and the adoption of workplace smoking policies. Health Education \& Behavior. 2000;27(4):483-501.

70. Glasgow RE, McCaul KD, Fisher KJ. Participation in worksite health promotion: a critique of the literature and recommendations for future practice. Health Education Quarterly. 1993;20(3):391-408.

71. Brownson RC, Hopkins DP, Wakefield MA. Effects of smoking restrictions in the workplace. Annual Review of Public Health. 2002;23(1):333-48.

72. Hopkins DP, Razi S, Leeks KD, Kalra GP, Chattopadhyay SK, Soler RE, et al. Smokefree policies to reduce tobacco use: a systematic review. American journal of preventive medicine. 2010;38(2):S275-S89.

73. Abrams DB, Niaura R. The tobacco dependence treatment handbook: A guide to best practices: Guilford Press; 2003.

74. Williams JM, Miskimen T, Minsky S, Cooperman NA, Miller M, Dooley Budsock P, et al. Increasing tobacco dependence treatment through continuing education training for behavioral health 
professionals. Psychiatric Services. 2014;66(1):21-6.

75. Fixsen DL, Naoom SF, Blase KA, Friedman RM. Implementation research: a synthesis of the literature. 2005.

76. Damschroder LJ, Reardon CM, Sperber N, Robinson CH, Fickel JJ, Oddone EZ. Implementation evaluation of the telephone lifestyle coaching (TLC) program: organizational factors associated with successful implementation. Translational behavioral medicine. 2016;7(2):233-41.

77. Cohen DJ, Crabtree BF. Evaluative criteria for qualitative research in health care: controversies and recommendations. The Annals of Family Medicine. 2008;6(4):331-9.

\section{Figures}

Phase 1: Exploratory Sequential Design Needs Assessment /Pre-Implementation

Implementation strategy: Translational formative evaluation to guide program development

AlM 1: To assess \& explore site-specific contextual factors that might affect program uptake to guide program development, fit and prepare for implementation

Phase 2: Program Implementation Program Monitoring and Adaptation Implementation strategies: ongoing organizational preparation, consultations and implementation of EBPs

AIM 2: To monitor and adapt uptake of TTTF within LMHAs

Phase 3: Explanatory Sequential Design Post-Implementation

Program Evaluation and Improvement Implementation strategies: identifying and enacting mechanisms for sustainability AIM 3: To evaluate program outcomes and characterize processes influencing reach, effectiveness, adoption, implementation, and maintenance

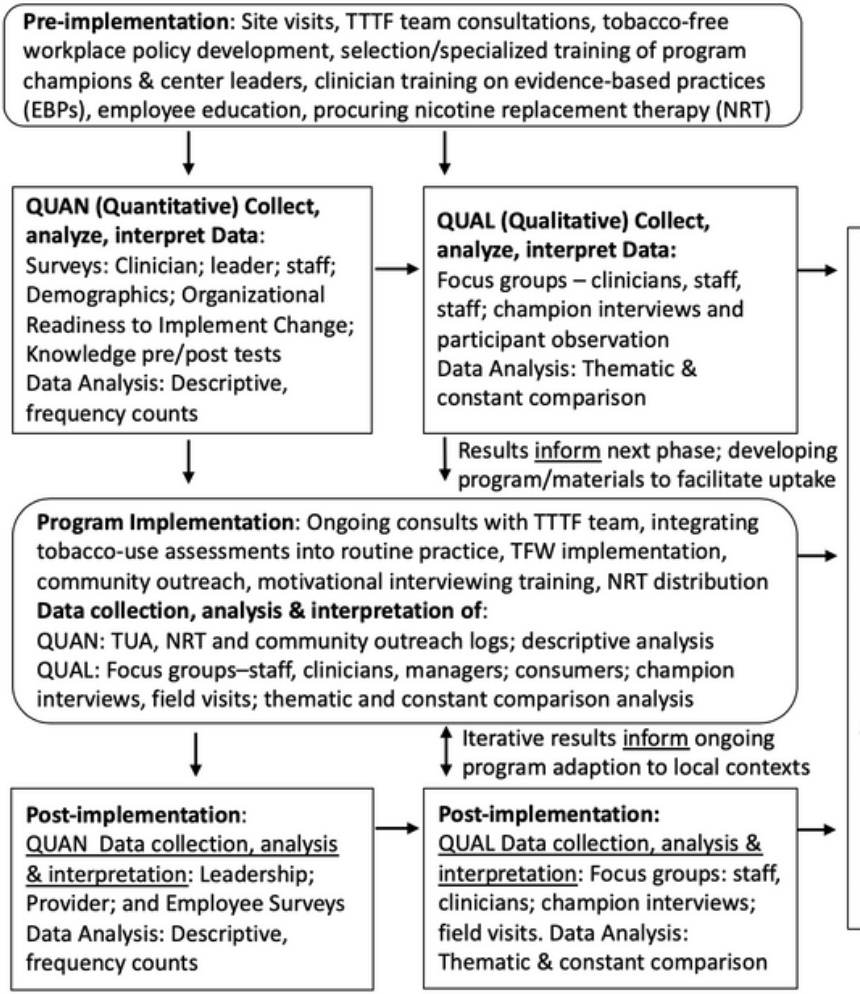

\section{Sites: $\mathbf{2}$ Local mental authorities (LMHAs)}

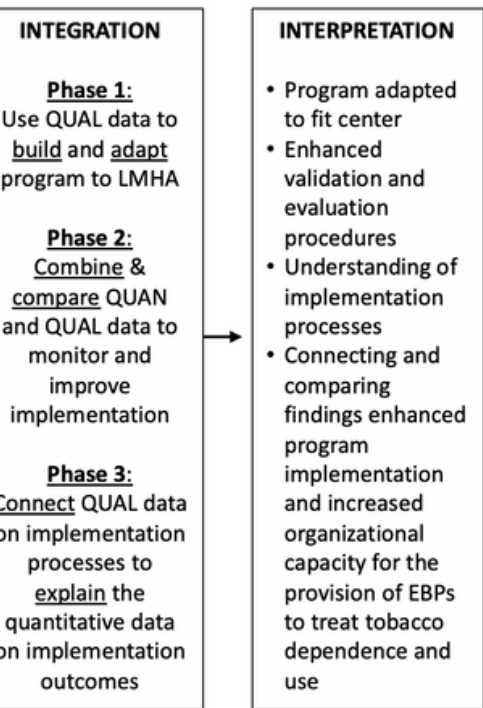

\section{Figure 1}

Mixed Methods Program Development and Evaluation Design of Taking Texas Tobacco Free (TTTF) 
Evidence-Based Education/Specialized Training of Clinicians and Staff

- General Staff: 1-hour interactive, live online (ZOOM) group training on tobacco use hazards, tobacco-free workplace (TFW) policies, use of FDA-approved medications (including nicotine replacement therapy (NRT), effects of nicotine on mental illness, myths regarding TFW programs and smoking among those with behavioral health conditions (BHCs)

- Clinicians: 2-hour interactive, live online (ZOOM) group training on all the above + evidence-based practices (EBPs) for treating tobacco dependence, delivering tobacco-use assessments (TUAs), behavioral counseling ( $5 \mathrm{~A}^{\prime}$ s model, motivational interviewing), and nicotine and drug interactions ITTF-sponsored special training of program champion and center implementation leaders - Designation and specialized group training (4-day Certified Tobacco Treatment Specialist training) of program champion to oversee program implementation

- Program champion and 3-5 center leaders receive specialized 2-day group training on treating tobacco dependence within behavioral health settings ${ }^{1}$

Develop, Implement and Enforce a 100\% Tobacco-Free Policy-Organization

- Banning all tobacco products (cigarettes, smokeless tobaccos, electronic nicotine systems)

- Designation of a tobacco-free date

- Installation of permanent signage publicly announcing tobacco-free policy

\section{Screening and Intervention-Clinicians and Organization}

- Embed regular tobacco use screenings (at each contact, weekly, or bi-monthly) into clinical practice

- Integrate EBPs for treating tobacco dependence (smoking counseling, TUAs and NRT therapies) into clinic to become widely and permanently available

\section{Community Engagement and Outreach-Organization}

- Assist local agencies to go tobacco-free

- Provide tobacco education and screenings

- Provide educational materials

1. Williams JM, Miskimen T, Minsky S, Cooperman NA, Miller M, Dooley Budsock P, et al. Increasing tobacco dependence treatment through continuing education training for behavioral health professionals. Psychiatric Services. 2014;66(1):21-6.
Barriers to Overcome

Clinician \& staff-level Barriers

- Clinicians lack training on

tobacco cessation treatment

- Misconceptions about tobacco use among those with BHCS

- Undervaluing tobacco addiction as a serious problem

- High tobacco use among employees and clinicians

- Low employee knowledge about tobacco addiction

Client-level Barriers

- Clients are not screened for tobacco use/dependence

- No advice or treatment for quitting tobacco provided for tobacco users

- Client High tobacco use rates

Community-level Barriers

- High rates of tobacco use in some communities (e.g. rural, low socio-economic status)

- Norms that tobacco use is common and not a serious health threat and addiction
Expected Goals

Clinician \& staff-leve

- Increase proportion of clinicians trained in tobacco cessation

- Increase quit attempts among employees and clinicians

- Increase proportion of employees educated about tobacco use

\section{Client-level}

- Increase tobacco-use assessments of clients

- Increase clinician provision of tobacco cessation treatment

- Increase quit attempts and decrease tobacco use among clients

\section{Community-level}

- Increase community tobacco education and outreach

\section{Figure 2}

Major Components of Taking Texas Tobacco Free and barriers targeted at behavioral health centers 